\title{
FACING UNIVERSITY'S SOCIAL RESPONSIBILITY: CONTRIBUTIONS FOR MINIMIZING THE RISK OF FOODBORNE LISTERIOSIS
}

\author{
Dupouy, E., Jechiu, T., Coşciug, L.
}

Technical University of Moldova, Department of Food Science and Nutrition

168 Stefan cel Mare blvd., Chisinau, MD-2004, Republic of Moldova

Correspondence: Eleonora Dupouy,E-mail: eleonora_dupouy@yahoo.com

\begin{abstract}
The worldwide emergence in recent years of Listeria monocytogenes as a food-borne pathogen of severe degree of danger implies raising awareness and food safety education enhancement as key strategies in preventing and minimizing the risk of listeriosis. Listeriosis is a rare but serious food-borne disease to which the immuno-compromised and the elderly are highly susceptible and at greater risk than the rest of the population. Pregnant women and neonates are subjects of particular health-injury and life-threatening implications. The educational programmes and campaigns for improving the knowledge on food-borne listeriosis have to focus on all actors involved into the food chain from production to consumption, meanwhile consumers' education being of high priority. The present study aims to contribute to the raising awareness regarding the risk of pregnant women's exposure to listeriosis. The study was a cross-sectional survey to assess food hygiene practices, food habits and the risk perception of listeriosis. Results show that only $5 \%$ of participants in the survey have heard about listeriosis. The study confirmed the hypothesis that Listeria as a food-borne pathogen is not known in the high-risk group of pregnant women. Educational sessions increased significantly the level of risk perception. The consumers' education paradigm aimed at preventing and reducing the risk of food-borne diseases is proposed.
\end{abstract}

Keywords: food-borne diseases, listeriosis, food safety, raising awareness

\section{Introduction}

Reducing the burden of food-borne diseases is one of the most stringent health problems worldwide. Addressing the mentioned problem is becoming even more important due to the food technologies advances with respective food chain prolongation and with the globalization of food market with foods exchanges and with respective increase of food-borne problems' spread to new areas (Ropkins and Beck, 2000; Van De Venter, 2000 ; Walls and Buchanan, 2005).

The available national statistics show that the sporadic cases and outbreaks in households are the main sources of food poisoning in Moldova that might be explained by the underestimation of possible health-threatening consequences caused by the food-borne pathogens. Salmonellosis are the most important infections. Data on non-identified and unknown pathogens are not available. Among other factors influencing the food-related hazards in Moldova are: limited access to information and knowledge on foodborne pathogens and respective low consumers' involvement in the decision-making process, consumers' high confidence in food suppliers, wide-spread commerce in the streets with ready-to-eat foods, occurrence on the market of falsified foods of illegal origin, inadequate refrigeration in the retail and homes, and food mishandling practices. Concerning the knowledge on Listeria and listeriosis the situation is even worst: dedicated pamphlets are not currently available and women are not informed about of listeriosis consequences.

\section{Methods}

Three questionnaires were developed by including questions relevant to the assessment of risk exposure and the access to information on food-borne diseases and listeriosis of the reference group: a) food frequency questionnaire on the consumption of 16 products at high-risk for 
listeriosis contamination; b) raw and cooked foods handling and storage and hygiene practices in food preparation; c) information sources and efficiency of risk communication.

Educational sessions, discussion and interviews with reference group sample were carried out using the developed pamphlet on Listeria, listeriosis and prevention-aimed practices. The reference group was represented by a sample of 40 pregnant women, including $30 \%$ inhabitants of the town and $70 \%$ - of the village. The age of the women participated in the study is comprised between 18 and 32, the level of education - $65 \%$ possess secondary and high school level studies and $35 \%$ have more advanced studies. The term of pregnancy is as follows: $10,3 \%-1^{\text {st }}$ trimester, $33,3 \%-2^{\text {nd }}$ trimester and $56,4 \%-$ in the $3^{\text {rd }}$ trimester.

\section{Results}

The obtained results show that the pregnant women are more often exposed at risk of listeriosis contamination through poor household hygiene practices and products mishandling, such as insufficient cooking of left-overs ( $45 \%$ of respondents), multiple re-heating of products $(18 \%)$, cross-contamination through the cutting boards and knives $(83 \%)$, and failure to use detergents when cleaning the work surfaces $(5 \%)$ and refrigerators $(68 \%)$. Also, many of interviewed women (55\%) do not pay attention when purchasing foods to the products' self-life. Only one person invoked concern in terms of food safety when purchasing her food. A number of respondents $(26 \%)$ claimed absence of knowledge on the recommended food during pregnancy, and the majority (58\%) do not believe that the consumption of certain foods could be harmful for the health of their foetus. Some interviewed women $(16 \%)$ think that harmful products might exist, but they do not know what these foods are. Concerning the usefulness of the provided information on Listeria and listeriosis - $69 \%$ have estimated it as very useful. $74 \%$ of respondents have expressed the intention to change the food choices by avoiding foods at high risk for Listeria contamination over the period of their pregnancy.

\section{Implications}

The carried study resulted in the raising awareness on Listeria and listeriosis of the participating pregnant women by providing knowledge on foods to be avoided during pregnancy and safer hygiene practices to be observed. There is hope that provided educational sessions and disscusion would be beneficial for the current and future pregnancies.

\section{Conclusions}

The study confirmed the hypothesis that Listeria as a food-borne pathogen is not known in the high-risk group of pregnant women. The educational campaigns are imperative. The university has to require more transparency by the improving reporting on food-borne illness, inclusive on listeriosis. It is important to promote the idea of setting a Center for Food-borne illness information and education attributed to develop educational database, printed and video materials for professionals and public; developing educational programmes and campaigns to update and improve knowledge on foodborne illness for industry, catering and consumers; fostering university's cooperation in the area of food-borne diseases prevention with other stakeholders.

\section{References}

1. Van De Venter T. (2000). Emerging food-borne diseases: a global responsibility. Food, Agriculture and Nutrition, Nr. 26, p. 4-13.

2. Ropkins K., Beck A.J. (2000). HACCP in the home: a framework for improving awareness of hygiene and safe handling with respect to chemical risk. Trends in Food Science \& Technology, 11, p. 105-114

3. Walls I., Buchanan R.L. (2005). Use of food safety objectives as a tool for reducing foodborne listeriosis. Food Control, 16 p. 795-799. 